\title{
Eliminación del ajuste por inflación a contribuyentes especiales del impuesto sobre la renta
}

\section{Effect of elimination of the adjustment for inflation to special taxpayers of the income tax}

\author{
४Jazmín Martínez \\ jazmin_martinezll@hotmail.com \\ ORCID: 0000-0001-6847-4016 \\ Universidad de Carabobo. Venezuela
}

\author{
\María Mejías \\ jazmin_martinezll@hotmail.com \\ ORCID: 0000-0001-6847-4016 \\ Universidad de Carabobo. Venezuela
}

Artículo recibido en julio 2019 / Arbitrado en agosto 2019 / Publicado en enero 2020

\section{RESUMEN}

El presente trabajo tiene como objetivo general, evaluar el efecto de la eliminación del sistema del Ajuste por Inflación de los Sujetos Pasivos Especiales como el caso de la empresa SERCOINFAL C.A en Valencia. Estado. Carabobo, Venezuela. Esta investigación estuvo enmarcada en la modalidad de investigación de campo, de tipo descriptiva y con apoyo documental. La muestra estudiada fue de cinco (05) empleados de la empresa mencionada, a quienes se aplicó una encuesta compuesta por quince (15) ítems, bajo una escala de Likert, De acuerdo a la información recolectada se concluye que los integrantes de la empresa no poseen conocimiento profundo sobre las obligaciones que deben cumplir, por otro lado, la Reforma Fiscal tiene efectos en la exclusión del Sistema del Ajuste por Inflación a los fines del Impuesto sobre la Renta, además interviene en la exclusión del enriquecimiento neto considerado para la presentación de Declaraciones Estimadas en el año fiscal, eliminación de la corrección monetaria o de la distorsión de la inflación sobre la base imponible del impuesto sobre la renta, degradación del cálculo de la renta imponible a valores históricos deformados por la inflación, entre otros.

Palabras clave: Ajuste por inflación, Sistema, contribuyentes especiales, impuesto sobre la renta, sujetos pasivos

The general objective of this work is to evaluate the effect of the elimination of the Inflation Adjustment system for Special Taxpayers, as in the case of the company SERCOINFAL C.A in Valencia. State. Carabobo, Venezuela. This research was framed in the field research modality, of a descriptive type and with documentary support. The sample studied was five (05) employees of the aforementioned company, to whom a survey composed of fifteen (15) items was applied, under a Likert scale.According to the information collected, it is concluded that the members of the company do not have in-depth knowledge of the obligations they must comply with. On the other hand, the Tax Reform has effects on the exclusion of the Inflation Adjustment System for the purposes of Income Tax, also intervenes in the exclusion of the net enrichment considered for the presentation of Estimated returns in the fiscal year, elimination of the monetary correction or inflation distortion on the income tax base, downgrading of the calculation of taxable income to historical values distorted by inflation, among others

Keywords: Adjustment for inflation, System, special taxpayers, income tax, taxpayers INTRODUCCIÓN 
En la actualidad la inflación es un hecho que ocurre en casi todos los países del mundo, en los subdesarrollados ocurre con mayor impacto ya que los índices de la misma, son más elevados afectando la economía de la población que habita en ellos, disminuyendo la calidad de vida de éstos; ya que el alza de los precios de los productos y servicios no es cubierta por los sueldos y salarios percibidos, es decir, ocurre una disminución del poder adquisitivo.

Por otro lado, se debe tener en cuenta que la designación de los llamados Sujetos Pasivos Especiales (SPE) se da por efecto de un acto administrativo del Servicio Nacional Integrado de Administración Aduanera y Tributaria (SENIAT), Venezuela, al cumplirse ciertos supuestos mínimos relativos al nivel de ingreso conforme lo prevé la normativa jurídica, pero tal calificación no es automática al lograrse dicho nivel de ingresos.

Es decir, ser un SPE no opera de pleno de derecho, sino que depende de la manifestación de voluntad del ente Administrativo una vez que se cumplen unos supuestos relativos a la cuantía de los ingresos brutos que esta debe verificar, quedando supeditado el régimen fiscal del Impuesto sobre la renta (ISLR), de un contribuyente, a un acto de la Administración Tributaria y no a un supuesto de Ley objetivo, con lo cual podría entenderse que se estaría vulnerando el principio de legalidad tributaria de rango constitucional. Esto ha creado grandes dudas por parte de los Contadores, ya que no entienden como podrán demostrar la inflación en el caso de las empresas al momento de realizar los estados financieros.

Por lo anterior expuesto se presenta este estudio que tuvo como objetivo, evaluar el efecto de la eliminación del sistema del ajuste por inflación de los sujetos pasivos especiales como el caso de la empresa SERCOINFAL C.A en Valencia. Estado Carabobo, Venezuela. De acuerdo a esto, se encuentra el caso de la empresa Servicio de Comedores Industriales Falcón (SERCOINFAL), líder en servicios de comedores industriales con más de 31 proyectos en los estados Anzoátegui, Aragua, Carabobo, Cojedes, Distrito Capital, Lara, Sucre y Táchira. En esta empresa, el contador externo realiza el sistema del Ajuste por Inflación, una vez que de la contabilidad se hayan corregido errores por omisión o desconocimiento que realiza las transacciones diariamente. Junto a la contabilidad se le envía los Estados de Situación Financiera, Activos Fijos, Estado de Resultados y Balance de Comprobación de todos los meses.

Hasta el momento, no han logrado actualizar la información que respecta el cambio del SENIAT y que consideran que puede verse afectada la organización al momento de realizar las declaraciones correspondientes al año en curso. Esta Reforma Fiscal tendrá efectos en la exclusión del Sistema del Ajuste por Inflación a los fines del Impuesto sobre la Renta, además interviene en la exclusión del enriquecimiento neto considerado para la presentación de Declaraciones Estimadas para el 2019, eliminación de la corrección monetaria o de la distorsión de la inflación sobre la base imponible del impuesto sobre la renta, degradación del cálculo de la renta imponible a valores históricos deformados por la inflación, entre otros.

\section{Sustento teórico del estudio}

A continuación, se presentan un conjunto de aspectos conceptuales que sirven de guía para la comprensión de la investigación; así mismo, permiten aclarar los términos que se estructuran partiendo de los aspectos generales a lo particular, con el propósito de ubicar al lector con el tema planteado y la ubicación de este con las áreas de conocimiento. 
Todas las organizaciones dedicadas a la producción o comercialización de bienes buscan la manera de implementar de forma óptima sus políticas para que los constantes cambios que el mundo experimenta no sean significativos; de esta premisa no escapan las empresas pequeñas $y$ medianas que ejercen sus actividades en el mundo, quienes tratan de alcanzar el éxito en un mercado altamente competitivo liderado por las grandes empresas, sólo quienes logren adaptarse y consigan superar los crecientes desafíos podrán consagrarse durante mucho tiempo con eficiencia.

Por otro lado, se encuentra la inflación, que es el incremento porcentual de los precios de una cesta de bienes y servicios involucrada en una realidad económica, entre dos períodos determinados. En efecto la inflación es un fenómeno económico que genera diversas consecuencias producida por el incremento general y constante en los precios de bienes y servicios que origina pérdida del poder adquisitivo, al disminuir progresivamente el valor real de la moneda.

Este fenómeno está presente en las economías de muchos países en el mundo, en algunas con mayor intensidad que en otras. La inflación afecta mayormente a las economías más susceptibles, principalmente la de países subdesarrollados, ya que son estos los que reciben mayor impacto a la hora de generarse el incremento acelerado y continúo de los precios de los productos, además son estos países los que son más vulnerables a sufrir devaluaciones monetarias, originándose de esta manera pérdida del poder adquisitivo.

Existen diversos factores que inciden en el origen de la inflación, entre los más comunes se encuentra la existencia de grandes cantidades de dinero en circulación que como consecuencia producen gran demanda, ocasionando que aumenten los costos de los productos de primera necesidad disminuyendo así el poder adquisitivo de los ciudadanos.

En tal sentido, se puede decir, que la inflación es la consecuencia de las políticas económicas ejecutadas por el gobierno nacional, debido a las malas planificaciones realizadas por los sectores económicos que rigen la materia en un determinado momento, siendo un fenómeno económico que afecta la situación financiera de las organizaciones, de acuerdo con Gómez y Leal (2009):

La inflación consiste en un
desequilibrio económico
caracterizado por el crecimiento
generalizado y continuo de los
precios de los bienes, servicios y
factores productivos de un país;
implica, por tanto, la reducción de
la capacidad adquisitiva del
dinero. Existen diversos factores
que la producen, sin embargo,
fundamentalmente se origina
cuando la oferta monetaria supera
la oferta de bienes y servicios.
(p.5l)

Lo anteriormente citado, no solo afecta a la población, también de manera directa a las empresas, especialmente a las medianas y pequeñas, ya que cada vez es más difícil lograr mantenerse en el mercado, debido al incremento en sus costos, los cuales son difíciles de cubrir, sobre todo, aquellas que se encuentren con los controles de precios $y$, además, hayan sido clasificadas como Sujetos Pasivos Especiales. En este orden de ideas se define la inflación de acuerdo a Faría y Sabino (2005:8). "la inflación, es un proceso económico caracterizado por alzas generalizadas y sostenidas de precios en el tiempo".

El incremento de la inflación en el caso de Venezuela ha sido incontrolado, los costos 
aumentan diariamente, lo que mantiene a los empresarios y compradores en permanente ajuste de precios, en el país, la inflación se mide mediante diferentes índices elaborados por el Banco Central de Venezuela, entre los que se encuentran: Índice de Precios al Consumidor, Índice de Precios al Mayor, Índice de Precios Nivel Productor.

Para medir el efecto que produce la inflación en las partidas no monetarias del activo y pasivo, se utilizan los sistemas del ajuste por Inflación que consisten en actualizar el valor histórico de los activos y pasivos no monetarios y a su vez, reconocer la pérdida o ganancia de los activos monetarios con los ajustes que se le realizan al patrimonio neto inicial. Existen dos tipos de ajustes por inflación: Los financieros y los Fiscales. Al respecto, Molina, V. (2006) señala que:

Ambas metodologías tienen objetivos similares, aunque para diferentes propósitos, pues para el ajuste financiero (DPC 10) la intención es reconocer el impacto que causa la inflación en la información financiera, es decir en los estados financieros básicos, balance general, ganancias y pérdidas, estado de movimiento de las cuentas patrimoniales y estado del flujo de efectivo. Mientras que el ajuste fiscal determina el impacto de la inflación en la renta del contribuyente, es decir en el enriquecimiento neto gravable. (p.61)

En esta investigación se utilizarán los ajustes fiscales, por ser estos los que contribuirán a desarrollar los objetivos establecidos en la investigación. Los sujetos que están obligados a realizar los ajustes por inflación están contenidos en la Ley de Impuesto Sobre La Renta. Artículo 173.

Ahora bien, los ajustes por inflación se deben presentar para mostrar la verdadera situación de la empresa en el momento de cuando se presenten los estados financieros, ya que debido a la inflación los estados financieros pueden estar presentando saldos no reales y por este motivo se deben actualizar dichos saldos, haciendo los ajustes correspondientes por los efectos de la inflación. Cabe considerar, que cuando un país está afectado por el proceso inflacionario, la contabilidad basada en costos históricos no responde a la realidad económica, debido a que las diferentes transacciones y operaciones están registradas con bolívares de diferente poder adquisitivo, por lo cual la información no es homogénea ni comparable.

Sin embargo, mediante el Decreto del gobierno venezolano, con rango, valor y fuerza de Ley Nro. 2.163 publicado en la Gaceta Oficial Nro. 6210 Extraordinario de fecha 30 de diciembre de 2015, el presidente de la República habilitado excepcionalmente para legislar, reforma nuevamente el marco jurídico que rige la determinación del Impuesto sobre la Renta. Dentro de los diferentes aspectos que se concretan en el contenido del instrumento legal nos encontramos con la caprichosa exclusión que se hace a los Sujetos Pasivos Especiales o Contribuyentes Especiales del sistema del Ajuste por Inflación fiscal.

La exposición de motivos del Decreto señala expresamente que "la reforma parcial elimina el Sistema del Ajuste por Inflación Fiscal, el cual se ha constituido en un mecanismo de disminución injustificada del pago de impuesto", se señala además que la reforma de la norma legal busca "aportar mayor progresividad y equidad al sistema tributario".

Esta premisa parte de un error conceptual que en primer término denota un copioso desconocimiento de la finalidad del Sistema del Ajuste por Inflación fiscal, conocido también como API fiscal, el cual es un elemento fundamental que sirve para medir la capacidad 
contributiva real del sujeto pasivo del ISLR y en ese sentido, contradictoriamente se incurre luego en una flagrante violación a los principios de progresividad y justicia tributaria, así como otros previstos en el artículo 316 del texto Constitucional.

Sin embargo, en una economía altamente inflacionaria, esta eliminación del ajuste impide a las empresas de servicios y otras con posiciones monetarias activas, deducir la perdida que supone la inflación en sus estructuras de financiamiento corporativo. Igualmente, le impide a la administración tributaria recaudar el impuesto que recae sobre la ganancia monetaria que supone acudir al financiamiento de terceros (banca principalmente y proveedores), lo cual es una ventaja para estas, pues en inflación el financiar la operación con deuda va a constituir un lucrativo negocio, ahora sin tener que pagar impuesto sobre estas ganancias.

Pero los entes afectados negativamente serán los propietarios de activos que se protegen de inflación, por ejemplo, de activos inmobiliarios, quienes tendrán que calcular la ganancia por enajenación comparando precios de venta actualizados con costos históricos rezagados. Esto constituirá en estos ambientes inflacionarios un impuesto prácticamente al patrimonio. Adicionalmente, existen nuevas reglas de determinación de la renta estimada a ser incluida en las Declaraciones Estimadas del Impuesto Sobre la Renta del 2016 donde se establece que las empresas deben presentar la Declaración Estimada estimando la renta gravable como usualmente se ha aplicado, pero excluyendo el efecto del sistema del Ajuste por Inflación del año anterior.

Esto va a beneficiar a las empresas que generan ganancias monetarias por recurrir al endeudamiento como forma de financiamiento operativo y financiero, pero afecta a quienes generan pérdidas monetarias como consecuencia de la exposición a la inflación de sus estructuras de financiamiento, lo cual implica en cualquier caso un pago adelantado del impuesto sobre la renta, que estas empresas finalmente no generan. El problema, más grave además de adelantar un impuesto calculado sobre una base que el contribuyente muy posiblemente no genere, es que en Venezuela el SENIAT no devuelve los pagos de impuestos en exceso incrementando la recaudación con a través de exacciones que para muchos especialistas las consideran ilegales.

Se debe tener en cuenta que la designación de los llamados Sujetos Pasivos Especiales (SPE) se da por efecto de un acto administrativo del SENIAT al cumplirse ciertos supuestos mínimos relativos al nivel de ingreso conforme lo prevé la normativa jurídica, pero tal calificación no es automática al lograrse dicho nivel de ingresos.

Es decir, ser un SPE no opera de pleno derecho, sino que depende de la manifestación de voluntad del ente Administrativo una vez que se cumplen unos supuestos relativos a la cuantía de los ingresos brutos que esta debe verificar, quedando supeditado el régimen fiscal del ISLR de un contribuyente, a un acto de la Administración Tributaria y no a un supuesto de Ley objetivo, con lo cual podría entenderse que se estaría vulnerando el principio de legalidad tributaria de rango constitucional.

Refiriéndonos ahora al sistema tributario, Ruiz (2011:49) lo define como el "conjunto adherente, sistemático e interrelacionado de impuestos que rige en un país en un momento determinado en el que debe cumplir ciertos requisitos para ser una estructura tributaria idónea". Los principios que regulan la tributación en Venezuela se encuentran consagrados en la vigente Constitución Nacional de la República Bolivariana de Venezuela (CNRBV, 1999), siendo 
los siguientes: Principio de Justa Distribución de las Cargas, Principio Pecuniario de la Obligación Tributaria y Principio de Igualdad y Generalidad.

Por otra parte, el Estado, como organización política, al coincidir sus fines con tales necesidades, asume su satisfacción como cometido estatal, tratándose de esa manera las mismas en necesidades públicas. Asegurar la previsión de los bienes y servicios que satisfagan tales necesidades constituye la más alta prioridad para el Estado, lo que lo vincula con el problema de financiamiento público, es decir, con la arbitración de mecanismos que garanticen el flujo de los recursos financieros necesarios para hacer frente a tal cometido. La única vía que le garantiza la certeza y en parte la suficiencia de recursos. A través de los tributos, se posibilita que sólo se emplace a contribuir para el financiamiento del gasto público a quien posea capacidad contributiva y sólo dentro del límite de la misma.

Por otra parte, el Impuesto Sobre la Renta es un impuesto, debido a que es un tributo cuya prestación es en dinero, exigidas por el Estado en virtud de su poder de imperio, a quienes se hallen en las situaciones consideradas por la ley como hecho imponible. Es un impuesto directo personal, según Rodríguez (2008:7), el impuesto directo es "el tributo o la obligación recae directamente sobre el sujeto que incurre en el hecho gravable, y no hay manera de poder trasladarlo a otra persona o sujeto. Es aquel impuesto que se paga al Fisco por el propio contribuyente", y el impuesto personal.

Según Moya (2003:192), "son aquellos impuestos en los que la determinación se efectúa con participación directa del contribuyente" y finalmente, es progresivo, ya que la alícuota aplicable aumenta en la medida que aumenta el monto gravado, fundamentado en el principio de justicia, es decir, que todos los ciudadanos de un país deben contribuir en proporción directa a su capacidad económica.

En resumen, el Impuesto sobre la Renta y en adelante ISLR, de acuerdo al Glosario de Tributos Internos (2008), publicado por el Servicio Nacional Integrado de Administración Aduanera y Tributaria (SENIAT) es un:

Impuesto aplicado sobre los
enriquecimientos anuales netos y
disponibles obtenidos en dinero o en
especie. Salvo disposición legal, toda
persona natural o jurídica, residente
o domiciliada en Venezuela, pagará
este impuesto sobre sus rentas de
cualquier origen, sea que la causa o la
fuente de ingreso esté situada dentro
del país o fuera de él. Las personas
naturales o jurídicas no residentes o
no domiciliadas en Venezuela,
estarán sujetas a este impuesto
siempre que la fuente o la causa de
sus enriquecimientos esté u ocurra
dentro del país, aun cuando no
tengan establecimiento permanente
o base fija en Venezuela, y en caso de
poseerlos, tributarán exclusivamente
por los ingresos de fuente nacional o
extranjera atribuibles a dicho
establecimiento permanente o base
fija. (p. 15)

El sistema de Ajuste por Inflación, fue incorporado por primera vez en Venezuela, en la reforma de la Ley de Impuesto Sobre la Renta en el año 1991, con el objeto de depurar la renta de los efectos distorsionantes de la inflación, la inclusión de esta metodología, constituye un reconocimiento legal a la incidencia que en la economía venezolana tiene el fenómeno de la inflación. Es la actualización anual, al cierre de cada ejercicio gravable, de los activos y pasivos no monetarios y del patrimonio neto al inicio, el cual generara un mayor o menor valor que será acumulado en una partida de conciliación fiscal 
denominada: Resistema del Ajuste por Inflación, el cual se tomara en consideración para la determinación de la renta gravable. Este sistema contempla dos tipos de ajustes, los cuales son: el ajuste inicial por inflación, que se realiza al cierre del primer ejercicio económicofiscal, y el ajuste regular por inflación, que debe realizarse consecuentemente, luego de cada cierre de ejercicio fiscal. El sistema del Ajuste por Inflación, es sólo a los fines de la aplicación de la Ley de 46 Impuesto Sobre la Renta, para la determinación de la renta gravable; en tal sentido, únicamente deberá practicarse al cierre de cada ejercicio tributario.

Rodríguez, J. (2010:294) señala el objetivo del sistema de ajuste integral por inflación fiscal es "depurar la renta de los efectos distorsionantes de la inflación"... "que la renta recaiga sobre la expresión real da la base imponible". Es decir, lo que persigue el sistema del Ajuste por Inflación fiscal es que se grave la renta realmente obtenida por el contribuyente, calculando el tributo sobre las ganancias reales. Ajuste Inicial por Inflación.

\section{MATERIALES Y METODOS}

De acuerdo con el propósito del estudio, el mismo se sustentó en una investigación de campo no experimental, de nivel evaluativo y de tipo descriptiva, con apoyo documental. Tratándose de un estudio de caso, se abordó la empresa SERCOINFAL C.A. ubicada en Valencia, Venezuela, a través de una muestra de cinco (5) integrantes de la misma (2 contadores y 3 analistas contables), a quienes se le aplicó un cuestionario conformado por quince (15) ítems, sistematizadas las respuestas a través de una escala de Likert, plasmando los resultados en cuadros de frecuencia relativa para su análisis.

En tal sentido, los datos se obtienen a través de instrumentos, de forma directa de la realidad con el propósito de describirlo, para efectos de explicar los efectos de la eliminación del sistema del Ajuste por Inflación en los Sujetos Pasivos Especiales, así como para entender su naturaleza y factores constituyentes, bajo el método de estudio de casos.

\section{RESULTADOS Y DISCUSION}

A continuación, se presentan los resultados más significativos de la investigación, producto de la aplicación del instrumento de recolección de datos, con la finalidad de dar respuesta al objetivo propuesto.

¿Está en conocimiento de la Reforma de la Ley de Impuesto sobre la Renta (LISLR, 2015) del 30 de diciembre del 2015 que extiende la exclusión del sistema del Ajuste por Inflación (API) a los sujetos pasivos calificados como especiales por la Administración Tributaria?

Tabla 1. Cumplimiento

\begin{tabular}{ccc}
\hline Escala & Frecuencia & Ítem 1 \\
\hline Totalmente de Acuerdo & 1 & $20 \%$ \\
De Acuerdo & 4 & $80 \%$ \\
Medianamente de Acuerdo & 0 & $0 \%$ \\
En Desacuerdo & 0 & $0 \%$ \\
Totalmente en Desacuerdo & 0 & $0 \%$ \\
Total & 5 & $100 \%$ \\
\hline
\end{tabular}

Fuente: Elaboración Propia (2019) 
Los resultados muestran que la gran mayoría de los empleados de la empresa están en conocimiento de la Reforma de la Ley de Impuesto sobre la Renta (LISLR, 2015) del 30 de diciembre del 2015, que extiende la exclusión del sistema de Sistema del Ajuste por Inflación (API) a los sujetos pasivos calificados como especiales por la Administración Tributaria. Esto es positivo, por cuento se debe tener conocimiento de dicha reforma, la cual permite, actualización de balances, el proyecto propone la incorporación de la normativa referente a un ajuste integral por actualización de los elementos del Activo, Pasivo y Patrimonio, con el propósito de que los sujetos pasivos especiales, paguen sobre la base de ingresos reales y no nominales como hasta el presente ha ocurrido.

En otra pregunta relacionada con el cumplimiento de las obligaciones relacionadas al
ISLR, la totalidad de los encuestados respondieron afirmativamente. Igualmente, cuando se preguntó a los encuestados si el pago del ISLR se realizan en los plazos correctos, el 100\% respondió estar totalmente de acuerdo, con lo cual se evitan sanciones y consecuencias que puedan afectar la productividad de la misma, acatando que el ISLR, es un tributo cuya prestación es en dinero, exigidas por el Estado en virtud de su poder de imperio, a quienes se hallen en las situaciones consideradas por la ley como hecho imponible.

¿Lleva de manera detallada los registros para presentar la declaración estimada calculando la renta gravable como usualmente se ha aplicado, pero excluyendo el efecto del sistema del Ajuste por Inflación del año anterior?

Tabla 2. Registros

\begin{tabular}{ccc}
\hline Escala & Frecuencia & Ítem 1 \\
\hline Totalmente de Acuerdo & 1 & $20 \%$ \\
De Acuerdo & 2 & $40 \%$ \\
Medianamente de Acuerdo & 2 & $40 \%$ \\
En Desacuerdo & 0 & $0 \%$ \\
Totalmente en Desacuerdo & 0 & $0 \%$ \\
Total & 5 & $100 \%$ \\
\hline
\end{tabular}

Fuente: Elaboración Propia (2019)

Con respecto a estos resultados, se puede decir que la contrapartida de las actualizaciones de los activos no monetarios y pasivos no monetarios, así como del patrimonio neto fiscal e inicial se registraran a los solos fines fiscales, en una partida de conciliación fiscal que se denominara, ajuste por inflación; y que se tomara en consideración a los efectos de la determinación de la renta gravable.

¿Considera usted que el sistema del Ajuste por Inflación, ayuda a reflejar la realidad económica y financiera de las empresas? 
Tabla 3. Sistema del Ajuste por Inflación

\begin{tabular}{ccc}
\hline Escala & Frecuencia & Ítem 1 \\
\hline Totalmente de Acuerdo & 0 & $0 \%$ \\
De Acuerdo & 0 & $0 \%$ \\
Medianamente de Acuerdo & 1 & $20 \%$ \\
En Desacuerdo & 2 & $40 \%$ \\
Totalmente en Desacuerdo & 2 & $40 \%$ \\
Total & 5 & $100 \%$ \\
\hline
\end{tabular}

Fuente: Elaboración Propia (2019)

El resultado arrojado, indica que los empleados encuestados, consideran que el sistema del Ajuste por Inflación, ayuda a reflejar la realidad económica y financiera de las empresas. Esto debido, a que, en materia impositiva, la inflación deforma o desvirtúa la definición de renta utilizada, debido a que la contabilidad se expresa en valores históricos y esto no muestra la realidad económica y financiera de los sujetos pasivos especiales.

Cuando se consultó a los encuestados acerca de su consideración acerca de los efectos negativos en la exclusión del enriquecimiento neto considerado para la presentación de Declaraciones Estimadas realizadas, la casi totalidad de los mismos manifestaros estar totalmente de acuerdo. Igualmente consideraron como injusta la exclusión del Sistema del Ajuste por Inflación a los Sujetos Pasivos Especiales. Sin embargo, la reforma mantiene la supresión del derecho a traspasar y compensar en ejercicios siguientes el saldo deudor del sistema del Ajuste por Inflación de aquellos contribuyentes obligados a realizar el ajuste integral por inflación (art. 181).

El sesenta por ciento (60\%) de los encuestados respondió casi siempre estar de acuerdo en que la inflación deforma la base imponible del Impuesto sobre la Renta, cuando esta medida sobre valores históricos.

¿Considera usted que esta reforma ocasiona pérdidas financieras a la empresa al no contemplar el Principio de Capacidad Contributiva y no Confiscatoriedad?

Tabla 4. Pérdidas

\begin{tabular}{ccc}
\hline Escala & Frecuencia & Ítem l \\
\hline Totalmente de Acuerdo & 3 & $60 \%$ \\
De Acuerdo & 2 & $40 \%$ \\
Medianamente de Acuerdo & 0 & $0 \%$ \\
En Desacuerdo & 0 & $0 \%$ \\
Totalmente en Desacuerdo & 0 & $0 \%$ \\
Total & 5 & $100 \%$ \\
\hline
\end{tabular}

Fuente: Elaboración Propia (2019) 
Por los resultados obtenidos, los empleados encuestados respondieron sesenta por ciento (60\%) siempre y cuarenta por ciento (40\%) casi siempre, considera usted que esta reforma ocasiona pérdidas financieras a la empresa al no contemplar el Principio de Capacidad Contributiva y no Confiscatoriedad.
¿Considera indispensable el Sistema del Ajuste por Inflación (API) para cuantificar de forma objetiva la capacidad contributiva y base para la determinación del ISLR del contribuyente?

Tabla 5. Ajustados

\begin{tabular}{ccc}
\hline Escala & Frecuencia & Ítem l \\
\hline Totalmente de Acuerdo & 4 & $80 \%$ \\
De Acuerdo & 1 & $20 \%$ \\
Medianamente de Acuerdo & 0 & $0 \%$ \\
En Desacuerdo & 0 & $0 \%$ \\
Totalmente en Desacuerdo & 0 & $0 \%$ \\
Total & 5 & $100 \%$ \\
\hline
\end{tabular}

Fuente: Elaboración Propia (2019)

En esta respuesta, se puede observar que el ochenta por ciento (80\%) respondió que siempre y veinte por ciento (20\%) casi siempre, considera indispensable el Sistema del Ajuste por Inflación (API) para cuantificar de forma objetiva la capacidad contributiva y base para la determinación del ISLR del contribuyente. Este sistema contempla dos tipos de ajustes, los cuales son: el ajuste inicial por inflación, que se realiza al cierre del primer ejercicio económico- fiscal, y el ajuste regular por inflación, que debe realizarse consecuentemente, luego de cada cierre de ejercicio fiscal.

Por lo anterior expuesto, tomando en consideración los resultados obtenidos, se presenta la siguiente matriz DOFA, en la cual se muestran los factores internos y externos que inciden en la gestión para el manejo del Sistema de Ajuste por Inflación de la empresa estudiada. 


\section{Cuadro 1. DOFA}

\begin{tabular}{|c|c|c|}
\hline & Lista de Fortalezas & Lista de Debilidades \\
\hline $\begin{array}{l}\text { FACTORES } \\
\text { INTERNOS }\end{array}$ & $\begin{array}{l}\text { F.1. Las obligaciones tributarias en materia de } \\
\text { ISLR son conocidas por parte del personal } \\
\text { administrativo y contable. } \\
\text { F.2. Se ejecutan las obligaciones tributarias de } \\
\text { la empresa. } \\
\text { F.3. Está en conocimiento de la Reforma de la } \\
\text { Ley de Impuesto sobre la Renta (LISLR, } \\
\text { 2015) del } 30 \text { de diciembre del } 2015 \text { que } \\
\text { extiende la exclusión del sistema de } \\
\text { Sistema del Ajuste por Inflación (API) a los } \\
\text { sujetos pasivos calificados como especiales } \\
\text { por la Administración Tributaria. } \\
\text { F.4. El pago del ISLR se realizan en los plazos } \\
\text { correctos. } \\
\text { F.5. Lleva de manera detallada los registros } \\
\text { para presentar la declaración estimada } \\
\text { calculando la renta gravable como } \\
\text { usualmente se ha aplicado pero } \\
\text { excluyendo el efecto del sistema del Ajuste } \\
\text { por Inflación del año anterior. }\end{array}$ & $\begin{array}{l}\text { D.1. La preparación de las declaraciones del } \\
\text { ISLR es no es realizada por una sola } \\
\text { persona. } \\
\text { D.2. No existir un manual de normas y y } \\
\text { procedimiento, para la gestión } \\
\text { tributaria. } \\
\text { D.3. La inflación, impacta negativamente en } \\
\text { la empresa. } \\
\text { D.4. Efectos negativos en la exclusión del } \\
\text { enriquecimiento neto considerado para } \\
\text { la presentación de Declaraciones } \\
\text { Estimadas realizadas en el 2016. } \\
\text { D.5. La reforma ocasiona pérdidas financieras } \\
\text { a la empresa al no contemplar el } \\
\text { Principio de Capacidad Contributiva y } \\
\text { no Confiscatoriedad. }\end{array}$ \\
\hline \multirow[t]{2}{*}{$\begin{array}{l}\text { FACTORES } \\
\text { EXTERNOS }\end{array}$} & Lista de Amenazas & Lista de Oportunidades \\
\hline & $\begin{array}{l}\text { A.1. Los cambios económicos que presentan el } \\
\text { país que dificultan las metas de la empresa. } \\
\text { A.2. Disminución de la inversión por parte del } \\
\text { sector privado y público. } \\
\text { A.3. La inflación y desabastecimiento afectan los } \\
\text { objetivos de la empresa }\end{array}$ & $\begin{array}{l}\text { O1. Posee una cartera importante de clientes } \\
\text { cautivos, lo que permita prever que las } \\
\text { mejoras propuestas contribuirán con la } \\
\text { expansión de sus operaciones. } \\
\text { O2. Lealtad de los clientes. } \\
\text { O.l. Excelente ubicación geográfica. }\end{array}$ \\
\hline
\end{tabular}

Fuente: Elaboración Propia (2019)

\section{CONCLUSIONES}

De acuerdo al análisis de los datos recolectados a través de los instrumentos aplicados, se puede apreciar que el efecto fundamental de la implementación del sistema de ajuste por inflación fiscal, fue tratar de sincerar la renta del contribuyente, y perseguir de tal forma, los aspectos reguladores tributarios como son los principios de capacidad contributiva y equidad. A tal efecto se estima recomendar a la empresa del caso de estudio, lo siguiente:

- Hacer de conocimiento general que además de las sanciones impuestas a la empresa, los autores de los ilícitos, así como los demás involucrados podrán ser sancionados si la administración tributaria demuestra su participación, asimismo hacer énfasis en los tipos de sanciones van desde multas hasta la inhabilitación para ejercicio de oficios y profesiones

- Elaborar un manual de normas y procedimientos para el área tributaria, especialmente con lo relacionado al impuesto sobre la renta, de esta manera todo el personal podrá instruirse sobre el tema.

- Delimitar las tareas fiscales a un personal en específico, asimismo ofrecer a dicho personal 
oportunidades de desarrollo profesional, mediante planificación y ejecución de planes de capacitación y adiestramiento tributarios, sin embargo, es importante destacar que los demás miembros del Área Contable de la empresa SERCOINFAL, C.A, a pesar de no estar directamente relacionados con las obligaciones tributarias de la empresa, es recomendable que posean información sobre este campo.

\section{REFERENCIAS}

Constitución de la República Bolivariana de Venezuela (1999). Gaceta Oficial de la República Bolivariana de Venezuela 36.860. Diciembre 30, 1999. Caracas, Venezuela

Faría Hugo J. y Sabino Carlos (2005). La Inflación que es y cómo eliminarla. Ed. CEDICE-Panapo, Caracas
Glosario de Tributos Internos (2008). Servicio Nacional Integrado de Administración Aduanera y Tributaria (SENIAT). www.seniat.gob.ve

Gómez, O. y Leal, M. (2009) Impacto del sistema del Ajuste por Inflación financiero en la gestión empresarial. Revista de Ciencias Sociales v.15 n.l Maracaibo mar. 2009

Moya, Edgar (2003). Elementos de Finanzas Públicas y Derecho Tributario. Editorial MobilLibros, 3era. Edición. Caracas. 169

Reglamento de la Ley de Impuesto Sobre la Renta (2003). Gaceta Oficial Extraordinaria de la República Bolivariana de Venezuela 5.662

Rodríguez, Jesús (2008). Curso Práctico de Impuesto Sobre La Renta. Editorial Corporación Marca, Caracas 